\title{
A combinatorial approach to surgery formulas in Heegaard Floer homology
}

\author{
EAMAN EFTEKHARY
}

\begin{abstract}
Using the combinatorial approach to Heegaard Floer homology, we obtain a relatively easy formula for computation of the groups $\widehat{\mathrm{HF}}\left(Y_{p / q}(K), \mathbb{Z} / 2 \mathbb{Z}\right)$, where $Y_{p / q}(K)$ is the three-manifold obtained by $p / q$-surgery on a knot $K$ inside a homology sphere $Y$.
\end{abstract}

57M27; 57R58

\section{Introduction}

In [3], the author used the combinatorial description of Heegaard Floer homology (see Sarkar and Wang [14] and Manolescu, Ozsváth and Sarkar [7] and also Manolescu, Ozsváth, Szabó and Thurston [8]) to obtain a gluing formula for Heegaard Floer homology, when two bordered three-manifolds with torus boundary are glued together. In this paper, we apply the gluing formula of [3] to the special case of rational surgeries on the knots inside homology spheres, and after simplifications, we derive a relatively easy formula for the Heegaard Floer homology of these three-manifolds.

Let $K$ be a knot inside the homology sphere $Y$. We may remove a tubular neighborhood of $K$ and glue it back in a different way to obtain the three-manifold $Y_{p / q}=Y_{p / q}(K)$, which is the result of $p / q$-surgery on $K$. The core of the solid torus, which is the tubular neighborhood of $K$, will represent a knot in $Y_{p / q}$ and will be denoted by $K_{p / q}$. We may denote $(Y, K)$ by $\left(Y_{\infty}, K_{\infty}\right)$, as an extension of the above notation. Let $\mathbb{H}_{\bullet}(K)$ be the Heegaard Floer homology group $\widehat{\operatorname{HFK}}\left(Y_{\bullet}, K_{\bullet}\right)$ for $\bullet \in \mathbb{Q} \cup\{\infty\}$. Note that $\widehat{\mathrm{HFK}}$ is defined for knots inside rational homology spheres (see Ozsváth and Szabó [10]) and that $\mathbb{H}_{0}(K)=\widehat{\mathrm{HFL}}(Y, K)$ is the longitude Floer homology of $K$ from the author's paper [6]. In all these cases, we choose the coefficient ring to be $\mathbb{Z} / 2 \mathbb{Z}$. If we choose a Heegaard diagram for $Y-K$ and let $\lambda_{\bullet}$ denote a longitude which has framing $\bullet \in \mathbb{Z} \cup\{\infty\}$ (with $\lambda_{\infty}=\mu$ the meridian for $K$ ), one can choose the curves $\lambda_{\bullet}$ so that the pairs $\left(\lambda_{\infty}, \lambda_{1}\right)$ and $\left(\lambda_{1}, \lambda_{0}\right)$ have a single intersection point in the Heegaard diagram. Let $(\bullet, \star) \in\{(\infty, 1),(1,0)\}$ correspond to either of these pairs. There are four quadrants around the intersection point of $\lambda_{\star}$ and $\lambda_{\bullet}$. If we 
puncture three of these quadrants and consider the corresponding holomorphic triangle map, we obtain an induced map $\mathbb{H}_{\bullet} \rightarrow \mathbb{H}_{\star}$. To construct this map, if

$$
H=\left(\Sigma, \boldsymbol{\alpha}=\left\{\alpha_{1}, \ldots, \alpha_{g}\right\}, \boldsymbol{\beta}_{0}=\left\{\beta_{1}, \ldots, \beta_{g-1}\right\}\right)
$$

is a Heegaard diagram for $Y-K$, one may set

$$
\boldsymbol{\beta}_{\star}=\left\{\beta_{1}^{\star}, \ldots, \beta_{g-1}^{\star}, \lambda_{\star}\right\},
$$

where $\beta_{i}^{\star}$ is an isotopic copy of the curve $\beta_{i}$. The triangle map is constructed from the pointed Heegaard triple

$$
\left(\Sigma, \boldsymbol{\alpha}, \boldsymbol{\beta}_{\bullet}, \boldsymbol{\beta}_{\star},\right. \text { three punctures). }
$$

If the punctures are chosen as in Figure 1, the result would be two maps $\phi, \bar{\phi}: \mathbb{H}_{\infty}(K) \rightarrow$ $\mathbb{H}_{1}(K)$ and two other maps $\psi, \bar{\psi}: \mathbb{H}_{1}(K) \rightarrow \mathbb{H}_{0}(K)$ so that the following two se-

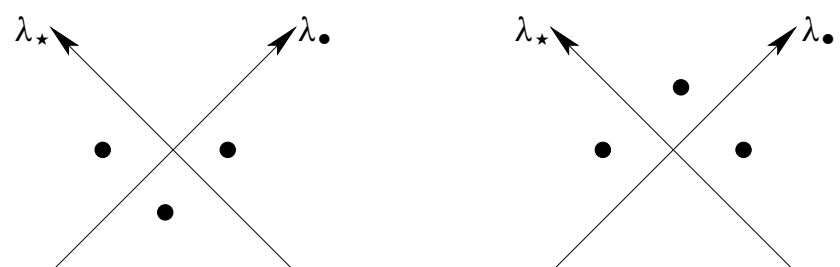

Figure 1: For defining chain maps between $C_{\bullet}(K)$ and $C_{\star}(K)$, the punctures around the intersection point of $\lambda_{\bullet}$ and $\lambda_{\star}$ should be chosen as illustrated in the above diagrams.

quences are exact (see Eftekhary [3, Lemma 3.2]):

and

$$
\begin{aligned}
& \mathbb{H}_{\infty}(K) \stackrel{\phi}{\rightarrow} \mathbb{H}_{1}(K) \stackrel{\bar{\psi}}{\rightarrow} \mathbb{H}_{0}(K) \\
& \mathbb{H}_{\infty}(K) \stackrel{\bar{\phi}}{\rightarrow} \mathbb{H}_{1}(K) \stackrel{\psi}{\rightarrow} \mathbb{H}_{0}(K)
\end{aligned}
$$

The homology of the mapping cones of $\phi$ (or $\bar{\phi}$ ) and $\psi$ (or $\bar{\psi}$ ) are $\mathbb{H}_{0}(K)$ and $\mathbb{H}_{\infty}(K)$ respectively [3, Section 3]. Let $\eta=\psi \circ \phi$ and $\bar{\eta}=\bar{\psi} \circ \bar{\phi}$. With the above notation fixed, we prove the following surgery formula:

Theorem 1.1 Let $K$ be a knot in a homology sphere $Y$ and let the complexes $\mathbb{H}_{\bullet}=$ $\mathbb{H}_{\bullet}(K), \bullet \in\{\infty, 1,0\}$ and the maps $\phi, \bar{\phi}, \psi, \bar{\psi}$ between them be as above. The homology of $Y_{p / q}(K)$, the manifold obtained by $p / q$-surgery on $K$ (for a pair of 
positive integers $p, q$ with $(p, q)=1)$, may be obtained as the homology of the complex $(\mathbb{H}, d)$ with

$$
\mathbb{H}=\left(\bigoplus_{i=1}^{q} \mathbb{H}_{\infty}(i)\right) \oplus\left(\bigoplus_{i=1}^{|p-q|} \mathbb{H}_{1}(i)\right) \oplus\left(\bigoplus_{i=1}^{p} \mathbb{H}_{0}(i)\right),
$$

where each $\mathbb{H}_{\bullet}(i)$ is a copy of $\mathbb{H}_{\bullet}$. Moreover, when $p \geq q$, the differential $d$ is the sum of the maps

$$
\begin{array}{cll}
\eta^{i}: \mathbb{H}_{\infty}(i) \rightarrow \mathbb{H}_{0}(i+p-q), & \bar{\eta}^{i}: \mathbb{H}_{\infty}(i) \rightarrow \mathbb{H}_{0}(i), & i=1,2, \ldots, q, \\
\psi^{j}: \mathbb{H}_{1}(j) \rightarrow \mathbb{H}_{0}(j), & \bar{\psi}^{j}: \mathbb{H}_{1}(j) \rightarrow \mathbb{H}_{0}(j+q), & j=1,2, \ldots, p-q,
\end{array}
$$

where $\psi^{i}$ is the map $\psi$ corresponding to the copy $\mathbb{H}_{1}(i)$ of $\mathbb{H}_{1}$, etc. Whenever $q>p$ the differential $d$ of the complex would be the sum of the maps

$$
\begin{array}{rlrl}
\eta^{i}: \mathbb{H}_{\infty}(i) \rightarrow \mathbb{H}_{0}(i+q-p), & \bar{\eta}^{i}: \mathbb{H}_{\infty}(i) \rightarrow \mathbb{H}_{0}(i), & i=1, \ldots, p, \\
\phi^{j}: & \mathbb{H}_{\infty}(j) \rightarrow \mathbb{H}_{1}(j), & \bar{\phi}^{j+q}: \mathbb{H}_{\infty}(j+q) \rightarrow \mathbb{H}_{1}(j), & j=1, \ldots, q-p .
\end{array}
$$

The surgery formulas given here do not make any reference to homological gradings, as they are derived from splicing formulas of the author [3], and homological grading is not discussed there. Working out the gradings in these two cases is an interesting problem on its own. The decompositions according to $\operatorname{Spin}^{c}$-structures are also not discussed. However, the investigations in the direction of finding a more explicit description of the maps $\phi, \bar{\phi}, \psi$ and $\bar{\psi}$ in the author's paper [4] results in understanding $\operatorname{Spin}^{c}$ decompositions. We thus refer the reader to [4] for this issue.

Surgery formulas for Heegaard Floer homology were first studied by Ozsváth and Szabó $[12 ; 10]$. Their work was generalized to a complete description of the quasiisomorphism type of the Heegaard Floer complex associated with $\left(Y_{n}(K), K_{n}\right)$ in terms of $\mathrm{CFK}^{\infty}(Y, K)$ by the author in [2]. The surgery formulas here are, however, simpler for actual computations. They are also used by the author in the proof of the main theorem of [1], which states that a prime homology sphere with trivial Heegaard Floer homology can not contain an incompressible torus. Moreover, in [5] we use these surgery formulas to show that if the rank of $\widehat{\operatorname{HFK}}(Y, K)$ for a knot $K$ inside a homology sphere $Y$ is the same as the rank of $\widehat{\mathrm{HF}}(Y)$, then $K$ is the unknot. This follows from a comparison of surgery formulas presented here with those of Ozsvath and Szabó [10].

Acknowledgements I would like to thank Matt Hedden for useful discussions which resulted in correcting a mistake in an earlier version of this paper. I would also like 
to thank the referee for many useful comments and suggestions, which resulted in a completely modified (and hopefully, easier to read) paper.

\section{A remark about splicing formulas}

Suppose that $K$ is a null-homologous knot inside a homology sphere $Y$. Let $\mathbb{H}_{\bullet}(K)$ be the group $\widehat{\operatorname{HFK}}\left(Y_{\bullet}, K_{\bullet} ; \mathbb{Z} / 2 \mathbb{Z}\right)$ introduced in the introduction for $\bullet \in\{0,1, \infty\}$. The maps $\phi, \bar{\phi}: \mathbb{H}_{\infty}(K) \rightarrow \mathbb{H}_{1}(K)$ and the maps $\psi, \bar{\psi}: \mathbb{H}_{1}(K) \rightarrow \mathbb{H}_{0}(K)$ are defined in [3] using a nice Heegaard diagram, ie a Heegaard triple in which all the regions, except one bad region, are bigons, rectangles or triangles. However, the definition of the maps given in the introduction does not make any reference to nice Heegaard diagrams. We will prove in this section that the definition of these maps is independent from the choice of the Heegaard diagram. We should check the independence of the definition, as given in the introduction, from the particular diagram, as we change the Heegaard diagram by isotopies, handle-slides, and stabilization (de-stabilization). We follow the general strategy of Ozsváth and Szabó in [11] (in particular Sections 8.3 and 8.4). Then we will show that for a nice diagram, the definition given in the introduction agrees with the one given by the combinatorial approach of [3].

Let us start the argument by fixing a Heegaard diagram

$$
H_{0}=\left(\Sigma, \boldsymbol{\alpha}=\left\{\alpha_{1}, \ldots, \alpha_{g}\right\}, \boldsymbol{\beta}_{0}=\left\{\beta_{1}, \ldots, \beta_{g-1}\right\}\right)
$$

for the knot complement $Y-K$. Set $\boldsymbol{\beta}_{\star}=\left\{\beta_{1}^{\star}, \ldots, \beta_{g-1}^{\star}, \lambda_{\star}\right\}$, where $\beta_{i}^{\star}$ is an isotopic copy of the curve $\beta_{i}$, and $\lambda_{\star}$ is chosen so that the Heegaard triple $\left(\Sigma, \boldsymbol{\alpha}, \boldsymbol{\beta}_{\star}\right)$ corresponds to the three-manifold obtained from $Y$ by $\star$-surgery on the knot $K$. Choose the curves $\lambda_{0}, \lambda_{1}$, and $\lambda_{\infty}$ so that each two of them have a unique transverse intersection point. The orientation on $K$ induces an orientation on the three curves $\lambda_{0}, \lambda_{1}$, and $\lambda_{\infty}$. Fix $\star, \bullet \in\{0,1, \infty\}$ and choose the marked points $u, v, w$ and $z$ on $\Sigma-\boldsymbol{\alpha}-\boldsymbol{\beta}_{\star}-\boldsymbol{\beta}_{\bullet}$ near the intersection point of $\lambda_{\star}$ and $\lambda_{\bullet}$ so that in Figure 1 the three punctures on the left-hand-side are $u, v$ and $w$, and the three punctures on the right-hand-side are $u, w$ and $z$. Note that the Heegaard diagrams $\left(\Sigma, \boldsymbol{\beta}_{\bullet}, \boldsymbol{\beta}_{\star} ; u, v, w\right)$, and $\left(\Sigma, \boldsymbol{\beta}_{\bullet}, \boldsymbol{\beta}_{\star} ; u, w, z\right)$ represent identical Heegaard Floer homology groups, which are both isomorphic to $\widehat{\mathrm{HF}}\left(\#^{g-1}\left(S^{1} \times S^{2}\right)\right)$, and the top generator $\Theta$ in this Heegaard Floer homology group may be used to define two holomorphic triangle maps.

Definition 2.1 For the Heegaard triple

$$
H_{\bullet, \star}=\left(\Sigma, \boldsymbol{\alpha}, \boldsymbol{\beta}_{\bullet}, \boldsymbol{\beta}_{\star} ; u, v, w, z\right)
$$


as above, define the maps

$$
\phi\left(H_{\bullet, \star}\right), \bar{\phi}\left(H_{\bullet, \star}\right): \widehat{\mathrm{HF}}\left(\Sigma, \boldsymbol{\alpha}, \boldsymbol{\beta}_{\bullet} ; u, w\right) \rightarrow \widehat{\mathrm{HF}}\left(\Sigma, \boldsymbol{\alpha}, \boldsymbol{\beta}_{\star} ; u, w\right)
$$

to be the holomorphic triangle maps corresponding to triply punctured Heegaard triples

$$
\left(\Sigma, \boldsymbol{\alpha}, \boldsymbol{\beta}_{\bullet}, \boldsymbol{\beta}_{\star} ; u, v, w\right) \&\left(\Sigma, \boldsymbol{\alpha}_{\bullet} \boldsymbol{\beta}_{\bullet}, \boldsymbol{\beta}_{\star} ; u, w, z\right),
$$

respectively, defined using the top generator $\Theta$ of $\widehat{\mathrm{HF}}\left(\Sigma, \boldsymbol{\beta}_{\bullet}, \boldsymbol{\beta}_{\star} ; u, w\right)$ (see Ozsváth and Szabó [11] for more details). Here $\widehat{\mathrm{HF}}(\Sigma, \boldsymbol{\alpha}, \boldsymbol{\beta} \bullet ; v, z)$ denotes the Heegaard Floer homology of the Heegaard diagram $\left(\Sigma, \boldsymbol{\alpha}, \boldsymbol{\beta}_{\bullet}\right)$, punctured at two points $u, w$ (hat theory). Let $\phi(K)=\phi\left(H_{\infty, 1}\right), \bar{\phi}(K)=\bar{\phi}\left(H_{\infty, 1}\right), \psi(K)=\phi\left(H_{1,0}\right)$ and $\bar{\psi}(K)=$ $\bar{\phi}\left(H_{1,0}\right)$.

The group $\widehat{\mathrm{HF}}\left(\Sigma, \boldsymbol{\alpha}, \boldsymbol{\beta}_{\bullet} ; v, z\right)$ is denoted by $\mathbb{H}_{\bullet}(K)$ in the introduction, and is independent of the particular Heegaard diagram used for the definition. We thus have defined the maps $\phi(K), \bar{\phi}(K): \mathbb{H}_{\infty}(K) \rightarrow \mathbb{H}_{1}(K)$ and $\psi(K), \bar{\psi}(K): \mathbb{H}_{1}(K) \rightarrow \mathbb{H}_{0}(K)$.

Theorem 2.2 The definitions of the maps $\phi(K), \bar{\phi}(K), \psi(K)$ and $\bar{\psi}(K)$ are independent from the choice of the particular Heegaard 4-tuple

$$
H=\left(\Sigma, \boldsymbol{\alpha}, \boldsymbol{\beta}_{\infty}, \boldsymbol{\beta}_{1}, \boldsymbol{\beta}_{0}\right) \text {. }
$$

More precisely, suppose that another Heegaard 4-tuple

$$
H^{\prime}=\left(\Sigma^{\prime}, \boldsymbol{\alpha}^{\prime}, \boldsymbol{\beta}_{\infty}^{\prime}, \boldsymbol{\beta}_{1}^{\prime}, \boldsymbol{\beta}_{0}^{\prime}\right)
$$

is given and $(\bullet, \star)$ with $\star, \bullet \in\{0,1, \infty\}$ is a pair of indices, and that $\{u, v, w, z\}$ and $\left\{u^{\prime}, v^{\prime}, w^{\prime}, z^{\prime}\right\}$ are the corresponding marked points for the two Heegaard triples $H_{\bullet}, \star$ and $H_{\bullet, \star}^{\prime}$ respectively. If the maps

$$
\begin{aligned}
& l_{\bullet}: \widehat{\mathrm{HF}}\left(\Sigma, \boldsymbol{\alpha}, \boldsymbol{\beta}_{\bullet} ; u, w\right) \rightarrow \widehat{\mathrm{HF}}\left(\Sigma^{\prime}, \boldsymbol{\alpha}^{\prime}, \boldsymbol{\beta}_{\bullet}^{\prime} ; u^{\prime}, w^{\prime}\right), \\
& l_{\star}: \widehat{\mathrm{HF}}\left(\Sigma, \boldsymbol{\alpha}, \boldsymbol{\beta}_{\star} ; u, w\right) \rightarrow \widehat{\mathrm{HF}}\left(\Sigma^{\prime}, \boldsymbol{\alpha}^{\prime}, \boldsymbol{\beta}_{\star}^{\prime} ; u^{\prime}, w^{\prime}\right)
\end{aligned}
$$

are the isomorphisms of the corresponding Heegaard Floer homology groups, we will have

$$
\phi\left(H_{\bullet, \star}\right) \circ l_{\bullet}=l_{\star} \circ \phi\left(H_{\bullet, \star}^{\prime}\right), \quad \text { and } \bar{\phi}\left(H_{\bullet, \star}\right) \circ l_{\bullet}=l_{\star} \circ \bar{\phi}\left(H_{\bullet, \star}^{\prime}\right) .
$$

Remark This theorem should be compared with the naturality theorem of Ozsvath and Stipsicz in [9]. 
In order to prove the above theorem, we should study what happens while Heegaard moves change one diagram to another. First of all, observe that once the Heegaard diagram $H_{0}=\left(\Sigma, \boldsymbol{\alpha}, \boldsymbol{\beta}_{0}\right)$ for the knot complement $Y-K$ and the pair of indices $\bullet, \star \in\{0,1, \infty\}$ are fixed, the two curves $\lambda_{\bullet}$ and $\lambda_{\star}$ are determined up to handle-slides of these two curves over the curves in $\boldsymbol{\beta}_{0}$, and isotopies away from $\boldsymbol{\beta}_{0}$ and the marked points. This observation, and the argument of [11] in Sections 8.3 and 8.4 imply the following proposition.

Proposition Suppose that the triples $H_{\bullet, \star}=\left(\Sigma, \boldsymbol{\alpha}, \boldsymbol{\beta}_{\bullet}, \boldsymbol{\beta}_{\star} ; u, v, w, z\right)$ and $H_{\bullet, \star}^{\prime}=$ $\left(\Sigma^{\prime}, \boldsymbol{\alpha}^{\prime}, \boldsymbol{\beta}_{\bullet}^{\prime}, \boldsymbol{\beta}_{\star}^{\prime} ; u^{\prime}, v^{\prime}, w^{\prime}, z^{\prime}\right)$ are two Heegaard diagrams as above corresponding to a knot $K \subset Y$ and a pair of indices $\bullet, \star \in\{0,1, \infty\}$. Then $H_{\bullet, \star}$ may be changed by a sequence of moves of the following types (and their inverses) to $H_{\bullet, \star}^{\prime}$.

- Handle slide of $\lambda_{\star}$ or $\lambda_{\bullet}$ over one of the curves in $\boldsymbol{\beta}_{0}$ (away from the marked points).

- Handle slide of one of the curves in $\boldsymbol{\beta}_{0}$ over another one of the curves in $\boldsymbol{\beta}_{0}$ (away from the marked points).

- Handle slide of one of the curves in $\boldsymbol{\alpha}$ over another one of the curves in $\boldsymbol{\alpha}$ (away from the marked points).

- Stabilization (and de-stabilization): taking the connected sum of $\Sigma$ with a standard torus, and adding the corresponding pair of curves to $\boldsymbol{\alpha}$ and $\boldsymbol{\beta}_{0}$.

- Isotopies of the Heegaard diagram, away from the marked points.

We will thus prove the previous theorem based on this proposition.

Proof of Theorem 2.2 It suffices to prove the theorem when the second diagram is obtained from the first diagram by a single move of the types described in the Proposition. Choose a pair of indices $\bullet, \star \in\{0,1, \infty\}$. For simplicity let $\bullet=\infty$ and $\star=1$. Let us first assume that $H_{\infty, 1}^{\prime}$ is obtained from $H_{\infty, 1}$ by a handle-slide of $\lambda_{\infty}$ over $\beta_{1}^{\infty}$. Let $\mu_{\infty}$ be the curve which is obtained from $\mu=\lambda_{\infty}$ by a handle-slide over $\beta_{1}^{\infty}$. Let us assume that the maps $\phi=\phi\left(H_{\infty, 1}\right)$ is defined as the triangle map associated with the Heegaard triple $\left(\Sigma, \boldsymbol{\alpha}, \boldsymbol{\beta}_{\infty}, \boldsymbol{\beta}_{1} ; u, v, w\right)$. We may choose $\mu_{\infty}$ so that it leaves in a small open neighborhood of $\lambda_{\infty} \cup \delta \cup \beta_{1}^{\infty}$, where $\delta$ is an arc in the complement of the curves in $\boldsymbol{\beta}_{\infty} \cup\left\{\lambda_{1}\right\}$ which connects $\lambda_{\infty}$ to $\beta_{1}^{\infty}$ and is used for the handle-slide. Let $\mu_{1}, \ldots, \mu_{g-1}$ denote isotopic copies of $\beta_{1}^{\infty}, \ldots, \beta_{g-1}^{\infty}$. The Heegaard triple $H_{\infty, 1}^{\prime}$ may be described by $\Sigma^{\prime}=\Sigma, \boldsymbol{\alpha}^{\prime}=\boldsymbol{\alpha}, \boldsymbol{\beta}_{\infty}^{\prime}=\boldsymbol{\mu}$ and $\boldsymbol{\beta}_{1}^{\prime}=\boldsymbol{\beta}_{1}$. Moreover, the marked points $u^{\prime}, v^{\prime}, w^{\prime}$ and $z^{\prime}$ may be assumed to be the same as $u, v, w$ and $z$. We thus obtain a Heegaard 4-tuple

$$
\left(\Sigma, \boldsymbol{\alpha}, \boldsymbol{\mu}=\left\{\mu_{1}, \ldots, \mu_{g-1}, \mu_{\infty}\right\}, \boldsymbol{\beta}_{\infty}, \boldsymbol{\beta}_{1} ; u, v, w\right),
$$


where the three punctures follow the model for $\phi$ in Figure 2. Note that the Heegaard

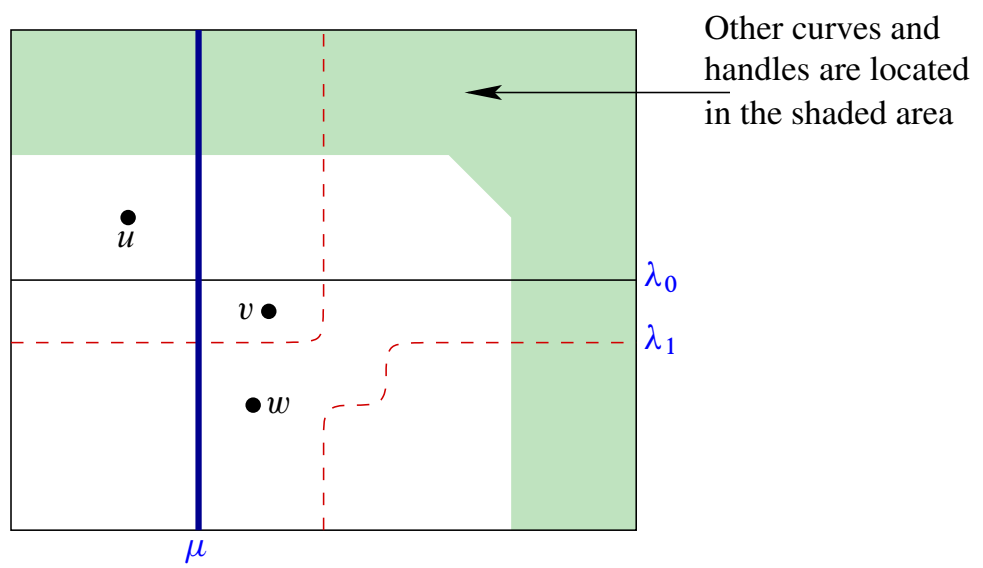

Figure 2: The Heegaard 4-tuple used for definition of the maps $\phi$ and $\bar{\psi}$

Floer homology group associated with the Heegaard diagram $\left(\Sigma, \boldsymbol{\mu}, \boldsymbol{\beta}_{\infty} ; u, v, w\right)$ is the Heegaard Floer homology of $\#^{g}\left(S^{1} \times S^{2}\right)$. Let $\Theta$ be the top generator of this homology group. The triple $\left(\Sigma, \boldsymbol{\alpha}, \boldsymbol{\mu}, \boldsymbol{\beta}_{\infty} ; u, v, w\right)$ gives a map

$\Phi: \widehat{\mathrm{HF}}(\Sigma, \boldsymbol{\alpha}, \boldsymbol{\mu} ; u, v, w) \otimes \widehat{\mathrm{HF}}\left(\Sigma, \boldsymbol{\mu}, \boldsymbol{\beta}_{\infty} ; u, v, w\right) \rightarrow \widehat{\mathrm{HF}}\left(\Sigma, \boldsymbol{\alpha}, \boldsymbol{\beta}_{\infty} ; u, v, w\right)$,

and we thus get a map $\Phi(., \Theta)$ from $\widehat{\mathrm{HF}}(\Sigma, \boldsymbol{\alpha}, \boldsymbol{\mu} ; u, v, w)$ to the Heegaard Floer group $\widehat{\mathrm{HF}}\left(\Sigma, \boldsymbol{\alpha}, \boldsymbol{\beta}_{\infty} ; u, v, w\right)$. Here $\widehat{\mathrm{HF}}$ of a Heegaard diagram is the Heegaard Floer homology associated with that punctured Heegaard diagram (ie we count the disks which miss the punctures). Note that $\Phi(., \Theta)$ is the natural isomorphism between the two homology groups $\widehat{\mathrm{HF}}(\Sigma, \boldsymbol{\alpha}, \boldsymbol{\mu} ; u, v, w)$ and $\widehat{\mathrm{HF}}\left(\Sigma, \boldsymbol{\alpha}, \boldsymbol{\beta}_{\infty} ; u, v, w\right)$ (which are both isomorphic to $\left.\mathbb{H}_{\infty}(K)\right)$. This isomorphism is the same map as $l_{\infty}^{-1}$ in the statement of the theorem. Similarly, we have a map

$\Psi: \widehat{\mathrm{HF}}\left(\Sigma, \boldsymbol{\alpha}, \boldsymbol{\beta}_{\infty} ; u, v, w\right) \otimes \widehat{\mathrm{HF}}\left(\Sigma, \boldsymbol{\beta}_{\infty}, \boldsymbol{\beta}_{1} ; u, v, w\right) \rightarrow \widehat{\mathrm{HF}}\left(\Sigma, \boldsymbol{\alpha}, \boldsymbol{\beta}_{1} ; u, v, w\right)$,

associated with the triple $\left(\Sigma, \boldsymbol{\alpha}, \boldsymbol{\beta}_{\infty}, \boldsymbol{\beta}_{1} ; u, v, w\right)$. Using the top generator $\Theta^{\prime}$ of $\widehat{\mathrm{HF}}\left(\Sigma, \boldsymbol{\beta}_{\infty}, \boldsymbol{\beta}_{1} ; u, v, w\right)=\widehat{\mathrm{HF}}\left(\#^{g-1}\left(S^{1} \times S^{2}\right)\right)$ we obtain a map $\Psi\left(., \Theta^{\prime}\right)$, which is precisely the map $\phi\left(H_{\infty, 1}\right)$ defined using the triple $\left(\Sigma, \boldsymbol{\alpha}, \boldsymbol{\beta}_{\infty}, \boldsymbol{\beta}_{1}\right)$. There are two other maps

$\Phi^{\prime}: \widehat{\mathrm{HF}}\left(\Sigma, \boldsymbol{\mu}, \boldsymbol{\beta}_{\infty} ; u, v, w\right) \otimes \widehat{\mathrm{HF}}\left(\Sigma, \boldsymbol{\beta}_{\infty}, \boldsymbol{\beta}_{1} ; u, v, w\right) \rightarrow \widehat{\mathrm{HF}}\left(\Sigma, \boldsymbol{\mu}, \boldsymbol{\beta}_{1} ; u, v, w\right)$,

$\Psi^{\prime}: \widehat{\mathrm{HF}}(\Sigma, \boldsymbol{\alpha}, \boldsymbol{\mu} ; u, v, w) \otimes \widehat{\mathrm{HF}}\left(\Sigma, \boldsymbol{\mu}, \boldsymbol{\beta}_{1} ; u, v, w\right) \rightarrow \widehat{\mathrm{HF}}\left(\Sigma, \boldsymbol{\alpha}, \boldsymbol{\beta}_{1} ; u, v, w\right)$, 
such that for any $a \in \widehat{\mathrm{HF}}(\Sigma, \boldsymbol{\alpha}, \boldsymbol{\mu} ; u, v, w)$ we have

$$
\Psi\left(\Phi(a \otimes \Theta) \otimes \Theta^{\prime}\right)=\Psi^{\prime}\left(a \otimes \Phi^{\prime}\left(\Theta \otimes \Theta^{\prime}\right)\right),
$$

by the associativity result of $\left[11\right.$, Section 8.4]. Note that $\Phi^{\prime}\left(\Theta \otimes \Theta^{\prime}\right)=\Theta^{\prime \prime}$, where $\Theta^{\prime \prime}$ is the top generator in $\widehat{\mathrm{HF}}\left(\Sigma, \boldsymbol{\mu}, \boldsymbol{\beta}_{1} ; u, v, w\right)=\widehat{\mathrm{HF}}\left(\#^{g-1}\left(S^{1} \times S^{2}\right)\right)$. Moreover, $\Psi^{\prime}\left(., \Theta^{\prime \prime}\right)$ is the map $\phi\left(H_{\infty, 1}^{\prime}\right)$ defined using the triple $\left(\Sigma, \boldsymbol{\alpha}, \boldsymbol{\mu}, \boldsymbol{\beta}_{1}\right)$. Since $l_{1}$ is the identity map, Equation (2-1) implies the claim of the theorem in this case. We may use the same argument to show the theorem when $\lambda_{1}$ is changed by a handle-slide, and more generally, to show the theorem once a different pair of indices $\star, \bullet \in\{0,1, \infty\}$ is used. The independence from handle slides among elements of $\boldsymbol{\alpha}$, and handle slides among elements of $\boldsymbol{\beta}_{0}$ (as well as independence from isotopies) is completely similar. Let us now suppose that $H_{\bullet, \star}^{\prime}$ is obtained from $H_{\bullet, \star}$ by stabilization. Suppose that the Heegaard diagram

$$
\left(\Sigma^{\prime}=\Sigma \# T, \boldsymbol{\alpha}^{\prime}=\boldsymbol{\alpha} \cup\left\{\alpha_{0}\right\}, \boldsymbol{\beta}_{\bullet}^{\prime}=\boldsymbol{\beta}_{\bullet} \cup\left\{\beta_{0}^{\bullet}\right\}, \boldsymbol{\beta}_{\star}^{\prime}=\boldsymbol{\beta}_{\star} \cup\left\{\beta_{0}^{\star}\right\}, u, v, w, z\right)
$$

is obtained by taking the connected sum of the original Heegaard diagram with the genus-one Heegaard diagram $\left(T, \alpha_{0}, \beta_{0}^{\bullet}, \beta_{0}^{\star}\right)$, which is presented in Figure 3. Make the

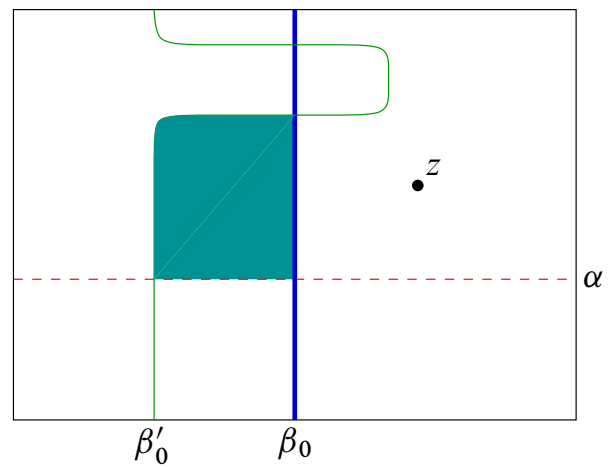

Figure 3: A Heegaard diagram $\left(T, \alpha=\alpha_{0}, \beta_{0}=\beta_{0}^{\infty}, \beta_{0}^{\bullet}=\beta_{0}^{\bullet}\right)$. The opposite sides of the rectangle glue together to give the surface $T$.

connected sum so that the domain containing the marked point $z$ in this later Heegaard diagram is glued to the domain containing one of the punctures. The Heegaard Floer complex associated with $(\Sigma, \boldsymbol{\alpha}, \boldsymbol{\beta} \bullet ; u, v, w)$ is naturally identified with the Heegaard Floer complex associated with $\left(\Sigma^{\prime}, \boldsymbol{\alpha}^{\prime}, \boldsymbol{\beta}_{\bullet}^{\prime} ; u, v, w\right)$. Similarly, the Heegaard Floer complex associated with $\left(\Sigma, \boldsymbol{\alpha}, \boldsymbol{\beta}_{\star} ; u, v, w\right)$ is naturally identified with the Heegaard Floer complex associated with $\left(\Sigma^{\prime}, \boldsymbol{\alpha}^{\prime}, \boldsymbol{\beta}_{\star}^{\prime} ; u, v, w\right)$. The domain of a holomorphic triangle for the Heegaard triple $\left(\Sigma^{\prime}, \boldsymbol{\alpha}^{\prime}, \boldsymbol{\beta}_{\bullet}^{\prime}, \boldsymbol{\beta}_{\star}^{\prime} ; u, v, w\right)$ consists of the domain of a 
holomorphic triangle in the old Heegaard triple plus a region on $T$ which is dashed in Figure 3. These two domains correspond to isomorphic moduli spaces of holomorphic triangles. Thus the map $\phi\left(H_{\bullet, \star}\right)$ is precisely the same as the map $\phi\left(H_{\bullet, \star}^{\prime}\right)$ under the above identification of the domain and the target. This proves the independence under stabilization (and destabilization), and completes the proof of the independence of the definition of $\phi\left(H_{\star}, \bullet\right)$ from the choice of Heegaard diagram. In particular, the maps $\phi(K), \bar{\phi}(K), \psi(K)$ and $\bar{\psi}(K)$ only depend on the knot $K$, and not on the particular Heegaard data. The proof of the theorem for the maps $\bar{\phi}\left(H_{\star, \bullet}\right)$ is identical.

We devote the last part of this section to showing that the map $\phi=\phi(K)$, as defined in the introduction, is the same as the map defined in [3] combinatorially (which will be denoted by $\phi^{\prime}$ ), provided that a particular Heegaard triple is used. We recall the definition of the map $\phi^{\prime}=\phi^{\prime}(K)$ for reader's convenience. See Figure 4.

This region contains 1-handles and complications of the intersection pattern

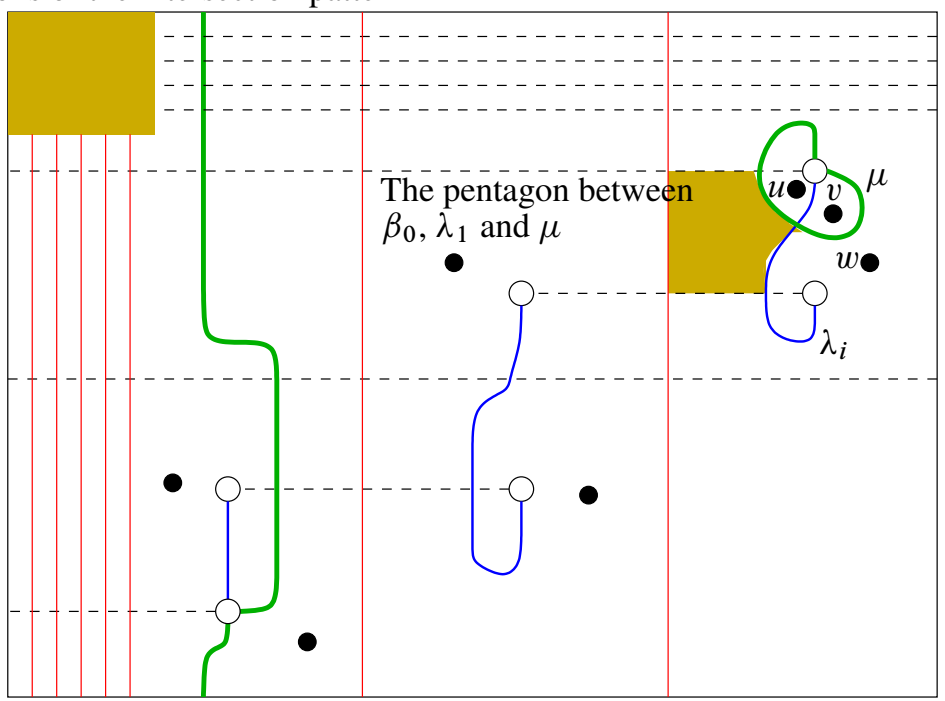

Figure 4: The Heegaard diagram used for defining $\phi^{\prime}$. Any pair of circles in the diagram denotes a 1 -handle. The red (solid) curves denote $\boldsymbol{\beta}_{0}$, the black (dashed) curves denote $\alpha$, the blue (solid) curve denotes $\lambda_{1}$, and the green (solid and bold) curve denotes $\mu=\lambda_{\infty}$. All the regions adjacent to the pentagon are punctured.

Again, we start with the Heegaard diagram

$$
H=\left(\Sigma, \boldsymbol{\alpha}=\left\{\alpha_{1}, \ldots, \alpha_{g}\right\}, \boldsymbol{\beta}_{0}=\left\{\beta_{1}, \ldots, \beta_{g-1}\right\}\right)
$$


for the complement of the knot $K \subset Y$, together with two additional curves $\beta_{g}=\lambda_{\infty}$ and $\lambda_{1}$ corresponding to the three-manifolds $Y$ and $Y_{1}(K)$ (the three-manifold obtained from $Y$ by 1 -surgery on $K$. Assume that three marked points $u, v$ and $w$ are chosen in the complement of $\boldsymbol{\alpha} \cup \boldsymbol{\beta}_{0} \cup\left\{\lambda_{\infty}, \lambda_{1}\right\}$, as before. Let

$$
\Sigma-\boldsymbol{\alpha}-\boldsymbol{\beta}_{0}-\left\{\lambda_{\infty}, \lambda_{1}\right\}=\left(\coprod_{i=1}^{N} D_{i}\right) \cup D_{u} \cup D_{v} \cup D_{w},
$$

where $D_{\bullet}$ are the regions in the complement of these curves, with $D_{u}, D_{v}$ and $D_{w}$ the regions containing the marked points. As it was the case in the construction of [3], let us assume that this data satisfies the following conditions:

(1) The regions $D_{2}, \ldots, D_{N}$ are either rectangles or bigons, while $D_{1}$ is a pentagon.

(2) One of the corners of the pentagon $D_{1}$ is the unique intersection point $P$ of $\lambda_{1}$ and $\beta_{g}=\lambda_{\infty}$, and the three marked points $u, v$ and $w$ are placed on three of the quadrants around $P$ (other than the quadrant corresponding to $D_{1}$ ).

(3) All the neighbors of $D_{1}$ (those regions having an edge in common with $D_{1}$ ) are punctured.

(4) Each $\beta$-curve is adjacent to at least one of the punctured domains.

The edges of the pentagon are five arcs: 2 of them are on $\lambda_{\infty}$ and $\lambda_{1}$, two of them are on $\alpha$-curves, and one of them is on a $\beta$-curve which will be assumed to be $\beta_{1}$. The $\alpha$-curve which cuts $\lambda_{\infty}$ in a corner of the pentagon will be assumed to be $\alpha_{1}$, and the other one will be assumed to be $\alpha_{2}$. Denote the vertices of the pentagon by $P=Q_{1}, Q_{2}, Q_{3}, Q_{8}$ and $Q_{6}$ in counterclockwise order, so that $Q_{1}$ is the intersection point of $\lambda_{\infty}$ and $\lambda_{1}, Q_{2}$ is on the intersection of $\alpha_{1}$ with $\lambda_{\infty}$, and so that $Q_{6}$ is the intersection point of $\lambda_{1}$ with $\alpha_{2}$. The strange labeling of these vertices will become clear in a moment (or the reader may check out Figure 6). Define the chain map

$$
\phi^{\prime}: \widehat{\mathrm{CF}}\left(\Sigma, \boldsymbol{\alpha}, \boldsymbol{\beta}_{0} \cup\left\{\lambda_{\infty}\right\} ; u, v, w\right) \rightarrow \widehat{\mathrm{CF}}\left(\Sigma, \boldsymbol{\alpha}, \boldsymbol{\beta}_{0} \cup\left\{\lambda_{1}\right\} ; u, v, w\right)
$$

by setting $\phi^{\prime}\left\{Q_{2}, Q_{8}, x_{3}, \ldots, x_{g}\right\}:=\left\{Q_{3}, Q_{6}, x_{3}, \ldots, x_{g}\right\}$, where $x_{i} \in \alpha_{i} \cap \beta_{\sigma(i)}$ for some $\sigma:\{3, \ldots, g\} \rightarrow\{1, \ldots, g-1\}$. For the rest of generators $\mathbf{x}$ of the left-hand group, set $\phi^{\prime}(\mathbf{x})=0$. It is shown in [3] that this is in fact a chain map, and induces a map

$$
\phi^{\prime}: \widehat{\mathrm{HF}}\left(\Sigma, \boldsymbol{\alpha}, \boldsymbol{\beta}_{0} \cup\left\{\lambda_{\infty}\right\} ; u, v, w\right) \rightarrow \widehat{\mathrm{HF}}\left(\Sigma, \boldsymbol{\alpha}, \boldsymbol{\beta}_{0} \cup\left\{\lambda_{1}\right\} ; u, v, w\right) .
$$

The fact that $\phi^{\prime}$ is a chain map may also be proved as a byproduct in the proof of our next theorem. We would like to compare this map with the map $\phi$ corresponding to an associated Heegaard triple. 
Let us construct a Heegaard triple from this data as follows. For $i=2, \ldots, g-1$, let $\mu_{i}$ be a parallel copy of $\beta_{i}$ which is drawn very close to $\beta_{i}$, and is slightly pushed to one of the punctured domains adjacent to $\beta_{i}$, so that a pair of intersection points (denoted by $p_{i}$ and $q_{i}$ ) is created between these two curves (see Figure 5). Let us

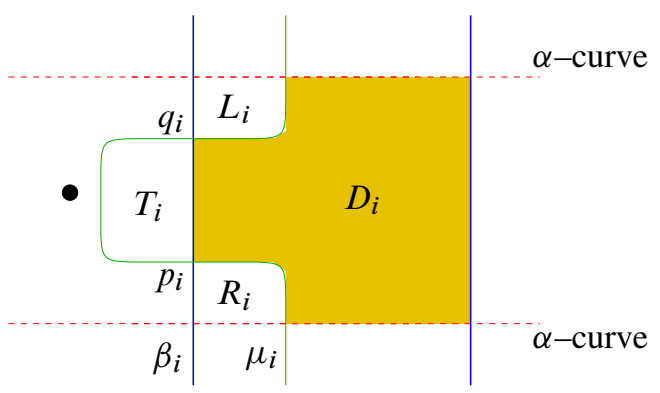

Figure 5: The diagram around the pair of intersection points between $\beta_{i}$ and $\mu_{i}$ for $i=2, \ldots, g-1$. The small bold circle denotes the punctured domain, and shaded region is the hexagon $D_{i}$.

assume that the small positively oriented disk connecting these two intersection points (with $\beta_{i}$ on the left and $\mu_{i}$ on the right) goes from $p_{i}$ to $q_{i}$. In order to define $\mu_{1}$, choose a parallel copy of $\beta_{1}$ and push it slightly over the intersection point of $\beta_{1}$ with $\alpha_{1}$ to obtain $\mu_{1}$, so that a pair of canceling intersection points $p_{1}$ and $q_{1}$ is created between $\mu_{1}$ and $\beta_{1}$ on the two sides of the intersection point $Q_{3}$ of $\alpha_{1}$ and $\beta_{1}$, and so that $\mu_{1}$ slightly enters the punctured domain next to the $\beta$-edge of the pentagon. The local picture around $D_{1}$ will look like Figure 6 where this procedure is pictured. Let $\mu_{g}$ be the curve $\lambda_{1}$ and set $\mu=\left\{\mu_{1}, \ldots, \mu_{g}\right\}$. The top generator $\Theta$ of the Heegaard Floer homology group $\widehat{\mathrm{HF}}\left(\#^{g-1} S^{1} \times S^{2}\right)$ coming from the Heegaard diagram $(\Sigma, \boldsymbol{\beta}, \boldsymbol{\mu}, u, v, w)$, is then the generator $\left\{P, p_{1}, \ldots, p_{g-1}\right\}$.

Theorem 2.3 If the surface $\Sigma$ and the sets of curves $\boldsymbol{\alpha}, \boldsymbol{\beta}_{0}$, and $\boldsymbol{\mu}$ and the curves $\lambda_{\infty}$ and $\lambda_{1}$ are as before, and if the homology group $\widehat{\mathrm{HF}}\left(\Sigma, \boldsymbol{\alpha}, \boldsymbol{\beta}_{0} \cup\left\{\lambda_{1}\right\} ; u, v, w\right)$ is identified with $\widehat{\mathrm{HF}}(\Sigma, \boldsymbol{\alpha}, \boldsymbol{\mu} ; u, v, w)$, the map $\phi(K)$ defined using the Heegaard triple

$$
H=(\Sigma, \boldsymbol{\alpha}, \boldsymbol{\beta}, \boldsymbol{\mu} ; u, v, w)
$$

and following Definition 2.1, is identical with the map $\phi^{\prime}$ defined using the Heegaard diagram $\left(\Sigma, \boldsymbol{\alpha}, \boldsymbol{\beta}_{0} \cup\left\{\lambda_{\infty}, \lambda_{1}\right\} ; u, v, w\right)$ as above.

Proof Let $\mathbf{x}=\left\{x_{1}, \ldots, x_{g}\right\}$ and $\mathbf{y}=\left\{y_{1}, \ldots, y_{g}\right\}$ be generators with $x_{i} \in \alpha_{\sigma(i)} \cap \beta_{i}$ and $y_{i} \in \alpha_{\tau(i)} \cap \mu_{i}$, where $\sigma, \tau:\{1, \ldots, g\} \rightarrow\{1, \ldots, g\}$ are permutations in $S_{g}$. Let $\Delta: \mathbb{D} \rightarrow \operatorname{Sym}^{g}(\Sigma)$ be the homotopy class of a triangle in $\pi_{2}(\mathbf{x}, \Theta, \mathbf{y})$, with Maslov 
index zero, such that it supports a holomorphic representative, and remains disjoint from the punctures. There are two types of domains in the complement $\Sigma-\boldsymbol{\alpha}-\boldsymbol{\beta}-\boldsymbol{\mu}$ of curves, the large domains and the small domain. The small domains are those created between the parallel pairs of curves $\mu_{i}$ and $\beta_{i}(i=1, \ldots, g-1)$, and their area may be chosen arbitrarily small by choosing $\mu_{i}$ close enough to $\beta_{i}$. The large domains are the rest of the domains which are in correspondence with the domains $D_{\bullet}, \bullet \in\{u, v, w, 0,1, \ldots, N\}$ introduced above. We will abuse the notation and will still denote these new regions by $D \bullet$.

Let us assume that the small bigon connecting $p_{i}$ to $q_{i}$ is denoted by $T_{i}$, and the region having the small interval $\left[p_{i}, q_{i}\right]$ on $\beta_{i}$ in common with $T_{i}$ is $D_{i}, i=2, \ldots, g-1$. Then there are two triangles with corners $p_{i}$ and $q_{i}$, which have an edge in common with $D_{i}$, which will be denoted by $R_{i}$ and $L_{i}$ respectively. For $i=1$, instead of these three regions we will have 4 triangles with one corner being $p_{1}$ or $q_{1}$, which will be denoted by $R_{1}, T_{1}, S_{1}$ and $L_{1}$ respectively (as they appear while we travel on $\beta_{1}$ from $p_{1}$ to $q_{1}$; see Figure 6). We are assuming that the regions $D_{i}$ for $i=1, \ldots, g-1$ (as described above) are different, while it may happen that this is not the case. However, the argument we give below will still be true in general, and only needs notational corrections. Let $\mathcal{D}=\mathcal{D}(\Delta)$ denote the domain (ie the 2-chain on $\Sigma$ ) associated with

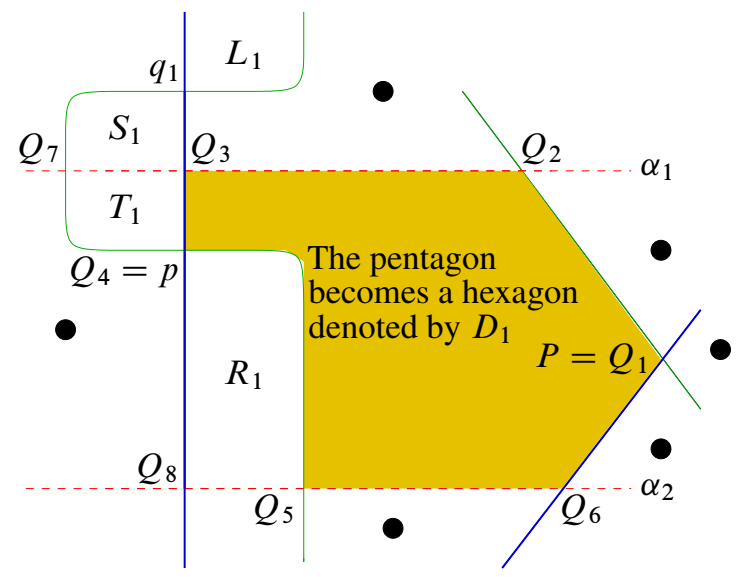

Figure 6: The regions around the pentagon $D_{1}$. The punctured domains are marked by a small solid circle inside them. The pentagon is changed to a hexagon in the new Heegaard diagram which is colored orange. The initial pentagon is the union of the hexagon $D_{1}$ with the triangle $R_{1}$.

this triangle map. Let $d_{i} \geq 0$ denote the coefficient of $D_{i}$ in $\mathcal{D}$. Similarly, denote the coefficients of $T_{i}, R_{i}$ and $L_{i}$ by $t_{i}, r_{i}$ and $l_{i}$ respectively. The coefficient of $S_{1}$ will be denoted by $s_{1}$. Of course, there are other regions which may appear in $\mathcal{D}$ with positive 
coefficient, but all such regions will be bigons or rectangles. Since $P$ appears in $\Theta$ and three of the corners around $P$ are punctured, the coefficient $d_{1}$ is equal to 1 . Let $P=Q_{1}, Q_{2}, \ldots, Q_{6}$ denote the corners of $D_{1}$ (now a hexagon) in counterclockwise order (so $Q_{4}=p_{1}$ ). Since two opposite quadrants around each one of $Q_{2}$ and $Q_{6}$ are punctured, we should have $x_{g}=Q_{2}$ and $y_{g}=Q_{6}$. From here, $Q_{3}$ may not be one of $x_{1}, \ldots, x_{g}$ and $Q_{5}$ may not be one of $y_{1}, \ldots, y_{g}$. Looking at the local coefficients around $Q_{3}$ we may thus conclude that $t_{1}=1+s_{1}$. If $Q_{7}$ is the third corner of $T_{1}$ (other than $Q_{3}$ and $Q_{4}$ ), in order for $\mathcal{D}$ to be a nonnegative domain, we should have $x_{1}=Q_{7}$, and the 4 local coefficients around $Q_{7}$ are forced to be $t_{1}=1+s_{1}, s_{1}, 0$ and 0 in the counterclockwise order. Two opposite quadrants around $q_{1}$ now have zero coefficients in $\mathcal{D}$. Since $q_{1}$ does not appear in $\Theta$, this implies that $s_{1}=l_{1}=0$ (thus $\left.t_{1}=1\right)$. Similarly, looking at the local coefficients around $p_{1}$, we should have $r_{1}=1$. Since $Q_{5}$ is not among $y_{1}, \ldots, y_{g}$, the local coefficients around $Q_{5}$ are $1, r_{1}=1,0$ and 0 in counterclockwise order. Let $Q_{8}$ be the third corner of $R_{1}$ other than $Q_{4}$ and $Q_{5}$. Since two opposite corners around $Q_{8}$ have zero coefficient, and $r_{1}=1$, we should have $x_{1}=Q_{8}$. This implies that $\mathcal{D}=\mathcal{D}^{\prime}+\mathcal{D}_{1}=\mathcal{D}^{\prime}+\left(R_{1}+D_{1}+T_{1}\right)$ where $\mathcal{D}^{\prime}$ is a nonnegative 2-chain which is disjoint from $\mathcal{D}_{1}$, and $\mathcal{D}_{1}$ is a hexagon with 5 acute angles and one obtuse angle, and with vertices $\left\{P, y_{g}, x_{1}, p_{1}, y_{1}, x_{g}\right\}$. The contribution of $\mathcal{D}_{1}$ to the index of $\Delta$ is zero, by Sarkar's formula from [13].

Note that by Sarkar's formula for the index of triangles from [13], the Maslov index $\mu(\Delta)$ is equal to

$$
e(\mathcal{D})+\mu_{\mathbf{x}}(\mathcal{D})+\mu_{\mathbf{y}}(\mathcal{D})+\mathfrak{c}(\mathcal{D}) \cdot \mathfrak{b}(\mathcal{D})-\frac{g}{2},
$$

where $e(\mathcal{D})$ is the Euler measure of the domain $\mathcal{D}, \mathfrak{b}(\mathcal{D})$ is the part of $\partial \mathcal{D}$ on the $\beta$-curves, and $\mathfrak{c}(\mathcal{D})$ is the part of $\partial \mathcal{D}$ on the $\mu$-curves. Separating $\mathcal{D}_{1}$, which has Maslov index 0 , from $\mathcal{D}$ we obtain the equality

$$
\mu(\Delta)=e\left(\mathcal{D}_{s}\right)+e\left(\mathcal{D}_{l}\right)+\mu_{\mathbf{x}}\left(\mathcal{D}^{\prime}\right)+\mu_{\mathbf{y}}\left(\mathcal{D}^{\prime}\right)+\mathfrak{c}\left(\mathcal{D}^{\prime}\right) . \mathfrak{b}\left(\mathcal{D}^{\prime}\right)-\frac{g-2}{2} .
$$

Here $\mathcal{D}_{s}$ denotes the part of $\mathcal{D}$ which uses the regions $D_{i}, R_{i}, T_{i}$ and $L_{i}$ for $i=$ $2, \ldots, g-1$ and $\mathcal{D}_{l}=\mathcal{D}^{\prime}-\mathcal{D}_{s}$. Clearly, $e\left(\mathcal{D}_{l}\right) \geq 0$ and

$$
\mathcal{D}_{s}=\sum_{i=2}^{g-1}\left(d_{i} D_{i}+t_{i} T_{i}+r_{i} R_{i}+l_{i} L_{i}\right) .
$$

By looking at the local coefficients around $p_{i}$ and $q_{i}$ it is easy to see that $r_{i}=l_{i}+1$ and $d_{i}=t_{i}+l_{i}$. Having in mind that $T_{i}$ are bigons, $R_{i}$ and $L_{i}$ are triangles and $D_{i}$ 
are hexagons, this implies the following computation:

$$
\begin{aligned}
e\left(\mathcal{D}_{s}\right) & =\sum_{i=2}^{g-1}\left(\left(t_{i}+l_{i}\right) e\left(D_{i}\right)+t_{i} e\left(T_{i}\right)+\left(l_{i}+1\right) e\left(R_{i}\right)+l_{i} e\left(L_{i}\right)\right) \\
& =\sum_{i=2}^{g-1}\left(\left(t_{i}+l_{i}\right)\left(-\frac{1}{2}\right)+t_{i}\left(\frac{1}{2}\right)+\left(l_{i}+1\right)\left(\frac{1}{4}\right)+l_{i}\left(\frac{1}{4}\right)\right)=\frac{g-2}{4} .
\end{aligned}
$$

The 1 -chain $\mathfrak{b}\left(\mathcal{D}^{\prime}\right)$ is a union of 1 -chains on $\beta_{i}, i=2, \ldots, g-1$, denoted by $\mathfrak{b}_{i}\left(\mathcal{D}^{\prime}\right)$. Similarly we have $\mathfrak{c}\left(\mathcal{D}^{\prime}\right)=\sum_{i=2}^{g-1} \mathfrak{c}_{i}\left(\mathcal{D}^{\prime}\right)$. It is clear that $\mathfrak{b}_{i}\left(\mathcal{D}^{\prime}\right)$ and $\mathfrak{c}_{j}\left(\mathcal{D}^{\prime}\right)$ are disjoint unless $i=j$. In this later case, the only possible geometric intersections are at $p_{i}$ and $q_{i}$, where the intersection numbers are $\left(l_{i}+\frac{1}{2}\right)\left(t_{i}-\frac{1}{2}\right)$ and $-l_{i} . t_{i}$ respectively. We thus have

$$
\mathfrak{c}\left(\mathcal{D}^{\prime}\right) \cdot \mathfrak{b}\left(\mathcal{D}^{\prime}\right)=\sum_{i=2}^{g-1}\left(\left(l_{i}+\frac{1}{2}\right)\left(t_{i}-\frac{1}{2}\right)-l_{i} \cdot t_{i}\right)=-\frac{g-2}{4}+\frac{1}{2} \sum_{i=2}^{g-1}\left(t_{i}-l_{i}\right)
$$

Let us now look at the coefficients around the intersection points $x_{i}$ and $y_{i}$ for $i=$ $2, \ldots, g-1$. Since $x_{i}$ is on $\beta_{i}$, we should have nonnegative integers $a_{i}, b_{i}, c_{i}$ and $e_{i}$ such that the local coefficients around $x_{i}$ are $a_{i}, b_{i}, b_{i}+l_{i}+1$ and $a_{i}+l_{i}$, and the local coefficients around $y_{i}$ are $c_{i}, e_{i}, e_{i}+t_{i}-1$ and $c_{i}+t_{i}$. From here we may compute

$$
\mu_{\mathbf{x}}\left(\mathcal{D}^{\prime}\right)+\mu_{\mathbf{y}}\left(\mathcal{D}^{\prime}\right)=\frac{1}{2} \sum_{i=2}^{g-1}\left(\left(a_{i}+b_{i}+c_{i}+e_{i}\right)+\left(l_{i}+t_{i}\right)\right) .
$$

Combining Equations (2-2), (2-3) and (2-4) and replacing for the terms in the definition of $\mu(\Delta)$ we obtain

$$
\begin{aligned}
0=\mu(\Delta) & =e\left(\mathcal{D}_{s}\right)+e\left(\mathcal{D}_{l}\right)+\mu_{\mathbf{x}}\left(\mathcal{D}^{\prime}\right)+\mu_{\mathbf{y}}\left(\mathcal{D}^{\prime}\right)+\mathfrak{c}\left(\mathcal{D}^{\prime}\right) \cdot \mathfrak{b}\left(\mathcal{D}^{\prime}\right)-\frac{g-2}{2} \\
& =e\left(\mathcal{D}_{l}\right)-\frac{g-2}{2}+\frac{1}{2} \sum_{i=2}^{g-1}\left(a_{i}+b_{i}+c_{i}+e_{i}+2 t_{i}\right) \\
& \geq \frac{1}{2} \sum_{i=2}^{g-1}\left(a_{i}+b_{i}+c_{i}+\left(e_{i}+t_{i}-1\right)+t_{i}\right) .
\end{aligned}
$$

Note that $e_{i}+t_{i}-1$ is the coefficient of one of the domains around $y_{i}$ and is thus nonnegative. The above inequality thus implies that $a_{i}=b_{i}=c_{i}=t_{i}=0$ and $e_{i}=1$ for $i=2, \ldots, g-1$. The coefficients on the two sides of $\mu_{i}$ will thus, either agree, or differ by 1 , and the coefficients on the two sides of $\beta_{i}$ differ either by $l_{i}$ or $l_{i}+1$. 
If we start from $y_{i}$, where on the left (or right) side of $y_{i}$ the coefficients on the two sides of $\mu_{i}$ are zero, and continue orthogonal to $\mu_{i}$ until we reach $\beta_{i}$, as we pass $\beta_{i}$ the coefficient should change either to $-l_{i}$ or to $-l_{i}-1$. Since the later is negative, the first one will happen and $l_{i}$ should be equal to 0 . It is easy to see from here that $x_{i}$ and $y_{i}$ should be the corresponding intersection points of $\beta_{i}$ and $\mu_{i}$ with the same $\alpha$ curve, and that the domain $\mathcal{D}^{\prime}$ is a union of obvious triangles which are disjoint from each other. We have concluded that the domain of $\Delta$ is the disjoint union of $g-2$ simple triangles with a hexagon with 5 acute angles and one obtuse angle. It is quite well-known that the moduli space corresponding to this homotopy class contributes 1 to the triangle map, for a generic path of almost complex structures. These are thus the only holomorphic triangles which contribute to the chain map $\phi$ defined using the Heegaard triple $(\Sigma, \boldsymbol{\alpha}, \boldsymbol{\beta}, \boldsymbol{\mu} ; u, v, w)$. Combinatorially, this is just the map, which replaces the pair $\left\{Q_{2}, Q_{8}\right\}$ with $\left\{Q_{6}, Q_{7}\right\}$. Under the obvious identification of $\widehat{\mathrm{HF}}(\Sigma, \boldsymbol{\alpha}, \boldsymbol{\mu} ; u, v, w)$ with $\widehat{\mathrm{HF}}\left(\Sigma, \boldsymbol{\alpha}, \boldsymbol{\beta}_{1} ; u, v, w\right)$ (obtained by choosing an appropriate complex structure for each Heegaard diagram), this is precisely the map $\phi^{\prime}$ considered in [3]. This completes the proof of our theorem.

The proof of a similar theorem for the maps $\bar{\phi}, \psi$ and $\bar{\psi}$ (and the corresponding combinatorial versions $\bar{\phi}^{\prime}, \psi^{\prime}$ and $\bar{\psi}^{\prime}$ ) is in fact easier, since the unpunctured domains in the Heegaard triple defining $\phi^{\prime}$ include a pentagon, together with a number of bigons and rectangles, while for the other maps we have a triangle instead of the pentagon. Working with a diagram containing a pentagon forces us, as was discussed in the proof of the above theorem, to describe carefully the domain associated to holomorphic triangles is a neighborhood of this pentagon. This part of the argument is not needed if the unpunctured domains do not have more than 4 edges.

\section{Surgery as splicing: a computation for the lens space}

Suppose that $p, q>0$ are relatively prime, and that $K$ is a null-homologous knot inside a three-manifold $Y$. The three-manifold $Y_{p / q}$ obtained as the result of $p / q-$ surgery on $K$ may also be realized as the outcome of splicing $K$ with a (rationally nullhomologous) knot $O_{p / q}$ inside the Lens space $L(p,-q)$. In fact, $L(p,-q)$ is obtained by gluing two solid tori along their boundary using an appropriate homeomorphism of their boundaries and in this situation $O_{p / q}$ is the core of one of the two pieces. Note that the appearance of $L(p,-q)$ instead of $L(p, q)$ serves to remain compatible with the splicing convention in [3]. This observation suggests that by computing the homology groups $\mathbb{H}_{\bullet}\left(O_{p / q}\right)$ for $\bullet \in\{0,1, \infty\}$ and the maps $\phi, \bar{\phi}: \mathbb{H}_{\infty}\left(O_{p / q}\right) \rightarrow \mathbb{H}_{1}\left(O_{p / q}\right)$ and $\psi, \bar{\psi}: \mathbb{H}_{1}\left(O_{p / q}\right) \rightarrow \mathbb{H}_{0}\left(O_{p / q}\right)$ we would have a surgery formula for knots inside three-manifolds. We will carry out this computation in the following two sections. 


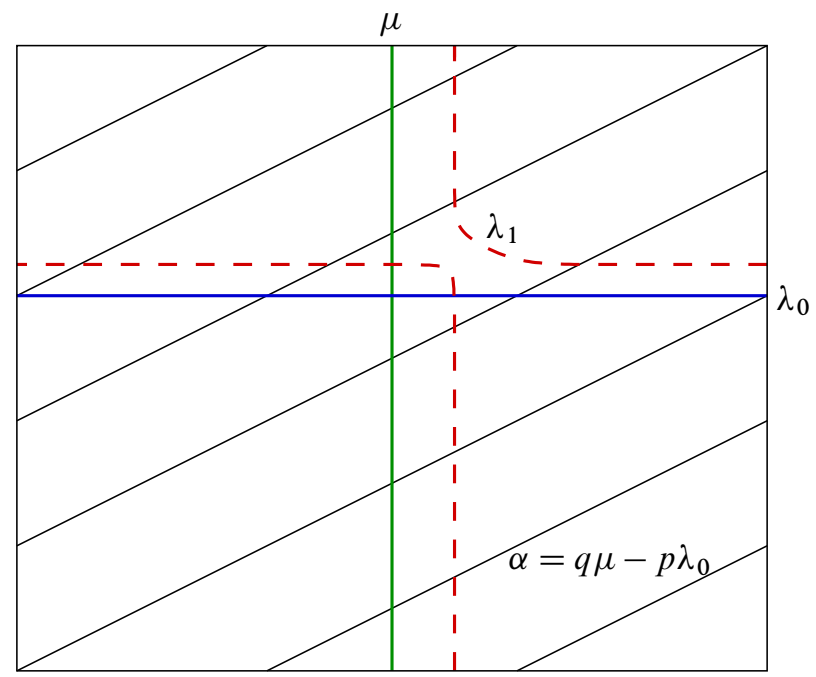

Figure 7: A Heegaard diagram $(\Sigma, \alpha, \mu)$ for $O_{p / q}$ together with two surgery curves $\lambda_{0}$ and $\lambda_{1}$

Consider the Heegaard diagram for $L(p,-q)$ which is illustrated in Figure 7. The surface $\Sigma$ in this Heegaard diagram is a genus 1 surface obtained by identification of the opposite sides of a $1 \times 1$ square. The curve $\alpha$ is the unique $\alpha$-curve, obtained as the image of a straight line with slope $q / p$ in the quotient surface. There are three other curves in this picture which are denoted by $\mu, \lambda_{0}$ and $\lambda_{1}$, and would give (together with $(\Sigma, \alpha))$ Heegaard diagrams for $L=L(p,-q), L_{0}\left(O_{p / q}\right)$ and $L_{1}\left(O_{p / q}\right)$ respectively. As before, $Y_{\ell}(K)$ is the three-manifold obtained from $Y$ by $\ell$-surgery on a framed knot $K$. There are $p, q$ and $p+q$ generators corresponding to the Heegaard diagrams $H_{\infty}=(\Sigma, \alpha, \mu), H_{0}=\left(\Sigma, \alpha, \lambda_{0}\right)$ and $H_{1}=\left(\Sigma, \alpha, \lambda_{1}\right)$ respectively. If we fix a marked point $z$ anywhere in the diagram, the $\operatorname{Spin}^{c}$ structures in $\operatorname{Spin}^{c}(L), \operatorname{Spin}^{c}\left(L_{0}\left(O_{p / q}\right)\right)$, and $\operatorname{Spin}^{c}\left(L_{1}\left(O_{p / q}\right)\right)$ assigned to the generators in each of these three sets are mutually different. This simply implies that

$$
\mathbb{H}_{\infty}\left(O_{p / q}\right)=(\mathbb{Z} / 2 \mathbb{Z})^{p}, \quad \mathbb{H}_{0}\left(O_{p / q}\right)=(\mathbb{Z} / 2 \mathbb{Z})^{q} \text {, and } \mathbb{H}_{1}\left(O_{p / q}\right)=(\mathbb{Z} / 2 \mathbb{Z})^{p+q} \text {. }
$$

We now compute the maps $\phi$ and $\bar{\phi}$ from $\mathbb{H}_{\infty}\left(O_{p / q}\right)$ to $\mathbb{H}_{1}\left(O_{p / q}\right)$. Let us denote the intersection points between $\alpha$ and $\mu$ by $x_{1}, \ldots, x_{p}$, and the intersection points between $\alpha$ and $\lambda_{1}$ by $y_{1}, \ldots, y_{p+q}$ as illustrated in Figure 8 . The unique intersection of $\mu$ and $\lambda_{1}$ is denoted by $M$. There are four marked points $u, v, w$ and $z$ in the four corners around $M$. Counting holomorphic triangles which miss the marked points in either of the Heegaard diagrams $H=\left(\Sigma, \alpha, \mu, \lambda_{1} ; u, v, w\right)$, and $\bar{H}=\left(\Sigma, \alpha, \mu, \lambda_{1} ; u, w, z\right)$ gives a map which would be $\phi$ and $\bar{\phi}$ respectively. It is easy to see that there is a unique 


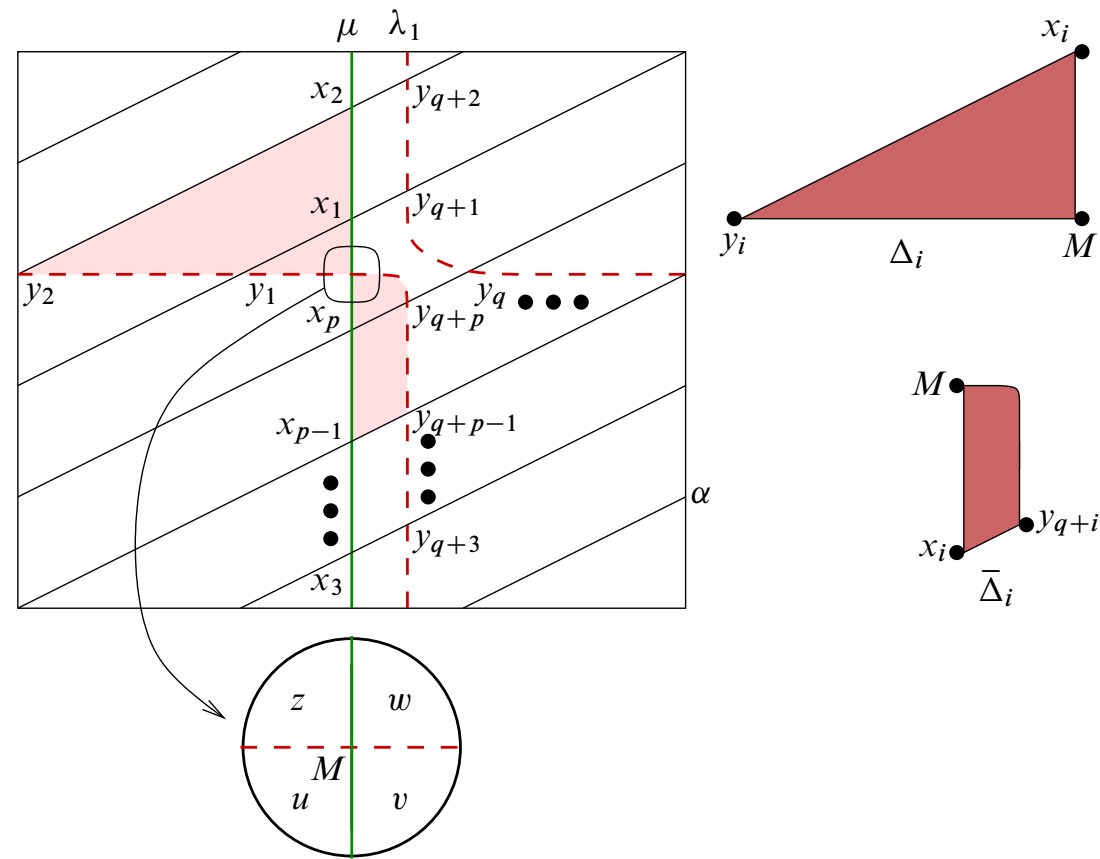

Figure 8: The diagram for the construction of the triangle maps $\phi$ and $\bar{\phi}$.

triangle $\Delta_{i}$ with vertices $\left(M, x_{i}, y_{i}\right)$ for $i=1,2, \ldots, p$ in the first diagram which is disjoint from the marked points $u, v, w$, and a triangle $\bar{\Delta}_{i}$ with vertices $\left(M, x_{i}, y_{i+q}\right)$ for $i=1,2, \ldots, p$ which is disjoint from $u, w, z$. Typical triangles are highlighted in Figure 8. It is not hard to see that these are all the relevant triangles. The maps $\phi$ and $\bar{\phi}$ will thus have the following matrix block forms as $p \times(p+q)$ matrices representing linear maps from $(\mathbb{Z} / 2 \mathbb{Z})^{p}$ to $(\mathbb{Z} / 2 \mathbb{Z})^{p+q}$ :

$$
\phi=\left(\begin{array}{ll}
I_{p \times p} & 0_{p \times q}
\end{array}\right), \quad \bar{\phi}=\left(\begin{array}{cc}
0_{p \times q} & I_{p \times p}
\end{array}\right) .
$$

Choosing an appropriate basis for $\mathbb{H}_{0}\left(O_{p / q}\right)$ (and the same basis for $\mathbb{H}_{1}\left(O_{p / q}\right)$ ) similarly gives the following matrix presentations of the maps $\psi$ and $\bar{\psi}$ :

$$
\psi=\left(\begin{array}{c}
I_{q \times q} \\
0_{p \times q}
\end{array}\right), \quad \bar{\psi}=\left(\begin{array}{c}
0_{p \times q} \\
I_{q \times q}
\end{array}\right) .
$$

\section{Obtaining surgery formulas from splicing formulas}

Suppose that $K_{1} \subset Y_{1}$ and $K_{2} \subset Y_{2}$ are two null-homologous knots inside threemanifolds $Y_{1}$ and $Y_{2}$. As before denote $\widehat{\mathrm{HF}}\left(\left(Y_{i}\right)_{\bullet}\left(K_{i}\right), K_{i} ; \mathbb{Z} / 2 \mathbb{Z}\right)$ by $\mathbb{H}_{\bullet}\left(K_{i}\right)$ where 
- is a surgery index in $\{0,1, \infty\}$, and $\left(Y_{i}\right)_{\bullet}\left(K_{i}\right)$ is the three-manifold obtained from $Y_{i}$ by $\bullet$-surgery on $K_{i}, i=1,2$. Let us denote $\mathbb{H}_{\bullet}\left(K_{1}\right) \otimes \mathbb{H}_{\star}\left(K_{2}\right)$ by $\mathbb{H}_{\bullet, \star}$ for $\bullet, \star \in\{0,1, \infty\}$. Let $\phi_{i}$ be the map $\phi\left(K_{i}\right)$ defined for the knot $K_{i} \subset Y_{i}$ for $i=1,2$. Similarly, define the maps $\bar{\phi}_{i}, \psi, \bar{\psi}_{i}, \eta_{i}$ and $\bar{\eta}_{i}$ for $i=1,2$, where $\eta_{i}=\psi_{i} \circ \phi_{i}$ and $\bar{\eta}_{i}=\bar{\psi}_{i} \circ \bar{\phi}_{i}$. Consider the complex

$$
\mathfrak{C} u=\left(\mathbb{H}_{\infty, \infty} \oplus \mathbb{H}_{1,1} \oplus \mathbb{H}_{1,0} \oplus \mathbb{H}_{0,1}\right) \bigoplus\left(\mathbb{H}_{1, \infty} \oplus \mathbb{H}_{\infty, 1} \oplus \mathbb{H}_{1,1} \oplus \mathbb{H}_{0,0}\right)
$$

with the differential $\mathfrak{D}$, which has the block form

$$
\mathfrak{D}=\left(\begin{array}{cc}
0 & \mathfrak{D}_{1} \\
\mathfrak{D}_{2} & 0
\end{array}\right)
$$

with $\mathfrak{D}_{1}$ and $\mathfrak{D}_{2}$ the following matrices respectively

$$
\left(\begin{array}{cccc}
I \otimes \phi_{2} & \phi_{1} \otimes I & 0 & \bar{\eta}_{1} \otimes \bar{\eta}_{2} \\
0 & 0 & 0 & 0 \\
0 & 0 & 0 & \psi_{1} \otimes I \\
0 & 0 & 0 & I \otimes \psi_{2}
\end{array}\right), \quad\left(\begin{array}{cccc}
0 & \phi_{1} \otimes I & \bar{\phi}_{1} \otimes \bar{\psi}_{2} & 0 \\
0 & I \otimes \phi_{2} & 0 & \bar{\psi}_{1} \otimes \bar{\phi}_{2} \\
0 & I & I \otimes \psi_{2} & \psi_{1} \otimes I \\
0 & 0 & 0 & 0
\end{array}\right) .
$$

The main theorem of [3] may be restated as follows.

Theorem 4.1 With the above notation, for the three-manifold $X$ obtained by splicing the knot complements $Y_{1}-K_{1}$ and $Y_{2}-K_{2}$, the Heegaard Floer homology group $\widehat{\mathrm{HF}}(X ; \mathbb{Z} / 2 \mathbb{Z})$ is isomorphic to the homology of the chain complex $(\mathfrak{C u}, \mathfrak{D})$.

This theorem, together with the observations of the previous section imply that the Heegaard Floer homology (hat theory) of $Y_{p / q}(K)$ is obtained as the homology of the complex $(\mathfrak{C} \mathfrak{d}, \mathfrak{D})$, where in the above setup $Y_{1}=Y, K_{1}=K, Y_{2}=L(p,-q)$ and $K_{2}=O_{p / q}$. From the computations of the previous section, we thus know

$$
\begin{array}{ll}
\phi_{2}=\left(\begin{array}{ll}
I_{p \times p} & 0_{p \times q}
\end{array}\right), & \bar{\phi}_{2}=\left(\begin{array}{cc}
0_{p \times q} & I_{p \times p}
\end{array}\right), \\
\psi_{2}=\left(\begin{array}{c}
I_{q \times q} \\
0_{p \times q}
\end{array}\right), & \bar{\psi}_{2}=\left(\begin{array}{c}
0_{p \times q} \\
I_{q \times q}
\end{array}\right) .
\end{array}
$$

To begin the simplifications, note that a particular change of basis for $\mathfrak{C} \mathfrak{u}$ may be described by a matrix of the form

$$
\mathfrak{P}=\left(\begin{array}{cc}
\mathfrak{P}_{1} & 0 \\
0 & \mathfrak{P}_{2}
\end{array}\right)
$$

and in the corresponding coordinates $\mathfrak{D}$ will be replaced with

$$
\mathfrak{D}(\mathfrak{P})=\left(\begin{array}{cc}
0 & \mathfrak{D}_{1}(\mathfrak{P}) \\
\mathfrak{D}_{2}(\mathfrak{P}) & 0
\end{array}\right)=\left(\begin{array}{cc}
0 & \mathfrak{P}_{1} \mathfrak{D}_{1} \mathfrak{P}_{2}^{-1} \\
\mathfrak{P}_{2} \mathfrak{D}_{2} \mathfrak{P}_{1}^{-1} & 0
\end{array}\right)
$$


In particular, if $\phi_{2}^{*}$ and $\psi_{2}^{*}$ are matrices with $\phi_{2} \phi_{2}^{*}=\operatorname{Id}$ and $\psi_{2}^{*} \psi_{2}=\mathrm{Id}$, we may choose $\mathfrak{P}_{1}$ and $\mathfrak{P}_{2}^{-1}$ to be the following two matrices respectively:

$$
\left(\begin{array}{cccc}
I & 0 & 0 & \bar{\eta}_{1} \otimes \bar{\eta}_{2} \psi_{2} \\
0 & I & I \otimes \psi_{2} & \psi_{1} \otimes I \\
0 & 0 & I & \psi_{1} \otimes \psi_{2}^{*} \\
0 & 0 & 0 & I
\end{array}\right), \quad\left(\begin{array}{cccc}
I & \phi_{1} \otimes \phi_{2}^{*} & \phi_{1} \otimes I & 0 \\
0 & I & I \otimes \phi_{2} & 0 \\
0 & 0 & I & 0 \\
0 & 0 & 0 & I
\end{array}\right) .
$$

Thus $\mathfrak{D}_{1}(\mathfrak{P})$ and $\mathfrak{D}_{2}(\mathfrak{P})$ will have the following matrix forms respectively:

$$
\left(\begin{array}{cccc}
I \otimes \phi_{2} & 0 & 0 & 0 \\
0 & 0 & 0 & 0 \\
0 & 0 & 0 & 0 \\
0 & 0 & 0 & I \otimes \psi_{2}
\end{array}\right), \quad\left(\begin{array}{cccc}
0 & 0 & \bar{\phi}_{1} \otimes \bar{\psi}_{2}+\phi_{1} \otimes \psi_{2}^{\prime} & \bar{\eta}_{1} \otimes I^{\prime} \\
0 & 0 & I \otimes \eta_{2} & \bar{\phi}_{2}+\psi_{1} \otimes \phi_{2}^{\prime} \\
0 & I & 0 & 0 \\
0 & 0 & 0 & 0
\end{array}\right) .
$$

Here $\psi_{2}^{\prime}=\left(I+\phi_{2}^{*} \phi_{2}\right) \psi_{2}, \phi_{2}^{\prime}=\phi_{2}\left(I+\psi_{2} \psi_{2}^{*}\right)$ and $I^{\prime}=\left(I+\phi_{2}^{*} \phi_{2}\right)\left(I+\psi_{2} \psi_{2}^{*}\right)$. Note that the first $p$ rows in $\bar{\psi}_{2}, \psi_{2}^{\prime}$ and $I^{\prime}$ and the first $q$ columns in $\bar{\phi}_{2}, \phi_{2}^{\prime}$ and $I^{\prime}$ are zero. In fact, assuming $p \geq q$ and $r=p-q, \psi_{2}^{\prime}, \phi_{2}^{\prime}$ and $I^{\prime}$ have the block forms

$$
\psi_{2}^{\prime}=0, \quad \phi_{2}^{\prime}=\left(\begin{array}{ccc}
0_{q \times q} & 0 & 0_{q \times q} \\
0 & I_{r \times r} & 0
\end{array}\right), \quad I^{\prime}=\left(\begin{array}{ccc}
0_{q \times q} & 0 & 0 \\
0 & 0_{r \times r} & 0 \\
0 & 0 & I_{q \times q}
\end{array}\right) .
$$

The homology of the complex with this differential is clearly the same as the homology of the complex

$$
\left(\mathbb{H}_{\infty} \otimes(\mathbb{Z} / 2 \mathbb{Z})^{q}\right) \oplus\left(\mathbb{H}_{1} \otimes(\mathbb{Z} / 2 \mathbb{Z})^{p}\right) \oplus\left(\mathbb{H}_{1} \otimes(\mathbb{Z} / 2 \mathbb{Z})^{q}\right) \oplus\left(\mathbb{H}_{0} \otimes(\mathbb{Z} / 2 \mathbb{Z})^{p}\right)
$$

with a differential which has the following matrix block form (assuming that $r=$ $p-q \geq 0)$ :

$$
\left(\begin{array}{cccc}
0 & 0 & \bar{\phi}_{1} \otimes I_{q \times q} & \eta_{1} \otimes\left(\begin{array}{ll}
0 & I_{q \times q}
\end{array}\right) \\
0 & 0 & I \otimes\left(\begin{array}{cc}
I_{q \times q} \\
0
\end{array}\right) & \bar{\psi}_{1} \otimes I_{p \times p}+\psi_{1} \otimes\left(\begin{array}{cc}
0 & 0 \\
I_{r \times r} & 0
\end{array}\right) \\
0 & 0 & 0 & 0 \\
0 & 0 & 0 & 0
\end{array}\right)
$$

Another change of coordinates, and a cancelation of $\mathbb{H}_{1} \otimes(\mathbb{Z} / 2 \mathbb{Z})^{q} \subset \mathbb{H}_{1} \otimes(\mathbb{Z} / 2 \mathbb{Z})^{p}$ in the second component of the above direct sum presentation against the third component using the map

$$
I \otimes\left(\begin{array}{c}
I_{q \times q} \\
0
\end{array}\right)
$$


implies that the homology of the initial complex is the same as the homology of the complex

$$
\mathbb{H}=\left(\mathbb{H}_{\infty} \otimes(\mathbb{Z} / 2 \mathbb{Z})^{q}\right) \oplus\left(\mathbb{H}_{1} \otimes(\mathbb{Z} / 2 \mathbb{Z})^{r}\right) \oplus\left(\mathbb{H}_{0} \otimes(\mathbb{Z} / 2 \mathbb{Z})^{p}\right)
$$

together with a differential which has the matrix block form

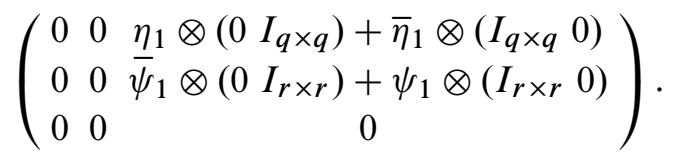

This computation completes the proof of Theorem 1.1 for $p \geq q$. The other case is completely similar.

\section{An example: the trefoil}

We finish this paper with a sample computation for the trefoils (leaving out many details). Let us consider the right-handed trefoil, whose filtration on $\widehat{\mathrm{CF}}\left(S^{3}\right)$ may be described as follows. The complex $\mathbb{B}=\widehat{\mathrm{CF}}\left(S^{3}\right)$ is generated by $x_{1}, x_{0}, x_{-1}$ with $\underline{\mathfrak{s}}\left(x_{i}\right)=i$ and the differential of the complex is given by $d\left(x_{0}\right)=x_{-1}$. Here $\underline{\mathfrak{s}}$ assigns relative $\operatorname{Spin}^{c}$ structures to the generators of the complex. Using the integral surgery formula of [2], we may compute $\mathbb{H} \bullet(s)$ for $\bullet \in\{0,1\}$ and $s \in \mathbb{Z}$ as the homology of a complex $C_{\bullet}(s)$. These complexes are given as the following mapping cones:

$$
\begin{aligned}
& C_{1}(s)=(\mathbb{B}\{\leq s\} \stackrel{\iota}{\rightarrow} \mathbb{B} \stackrel{\imath}{\leftarrow} \mathbb{B}\{\leq-s\}), \\
& C_{0}(s)=(\mathbb{B}\{\leq s\} \stackrel{\iota}{\rightarrow} \mathbb{B} \stackrel{\imath}{\leftarrow} \mathbb{B}\{\leq-s-1\}) .
\end{aligned}
$$

We may thus compute $\mathbb{H}_{1}(1)=\mathbb{H}_{1}(0)=\mathbb{H}_{1}(-1)=\mathbb{Z} / 2 \mathbb{Z}$ and $\mathbb{H}_{0}(0)=\mathbb{H}_{0}(-1)=$ $(\mathbb{Z} / 2 \mathbb{Z}) \oplus(\mathbb{Z} / 2 \mathbb{Z})$. Thus $\mathbb{H}_{\infty}=\mathbb{H}_{1}=(\mathbb{Z} / 2 \mathbb{Z})^{3}$ while $\mathbb{H}_{0}=(\mathbb{Z} / 2 \mathbb{Z})^{4}$. We may check, either by direct computation, or using the general formulation of the maps $\phi, \bar{\phi}, \psi$ and $\bar{\psi}$ given in [4] that these maps will have the following matrix presentations:

$$
\begin{aligned}
& \phi=\left(\begin{array}{lll}
1 & 0 & 0 \\
0 & 0 & 0 \\
0 & 0 & 0
\end{array}\right), \\
& \bar{\phi}=\left(\begin{array}{lll}
0 & 0 & 0 \\
0 & 0 & 0 \\
0 & 0 & 1
\end{array}\right) \\
& \psi=\left(\begin{array}{llll}
1 & 0 & 0 & 0 \\
0 & 1 & 0 & 0 \\
0 & 0 & 0 & 0
\end{array}\right), \\
& \bar{\psi}=\left(\begin{array}{llll}
0 & 0 & 0 & 0 \\
0 & 0 & 1 & 0 \\
0 & 0 & 0 & 1
\end{array}\right) .
\end{aligned}
$$


Thus the differential of the complex for $p \geq q$ is given by a matrix of the form

$$
\left(\begin{array}{ll}
0 & D \\
0 & 0
\end{array}\right)
$$

where $D$ is the following matrix

$$
D=\left(\begin{array}{cccccccc}
I_{r \times r} & 0_{r \times q} & 0 & 0 & 0 & 0 & 0 & 0 \\
0 & 0 & I_{r \times r} & 0_{r \times q} & 0_{r \times q} & I_{r \times r} & 0 & 0 \\
0 & 0 & 0 & 0 & 0 & 0 & 0_{r \times q} & I_{r \times r} \\
0 & I_{q \times q} & 0 & 0 & 0 & 0 & 0 & 0 \\
0_{q \times r} & 0 & 0 & 0 & 0 & 0 & 0 & 0 \\
0 & 0 & 0 & 0 & 0 & 0 & I_{q \times q} & 0
\end{array}\right) .
$$

This $(3 r+3 q) \times 4 p$ matrix is of rank $2 p+r$. So the rank of the homology group of the complex is $3(r+q)+4 p-2(2 p+r)=2 q+p$. If $q>p>0$, a similar computation gives the same rank $2 q+p$ for $\widehat{\mathrm{HF}}\left(S_{p / q}^{3}(T), \mathbb{Z} / 2 \mathbb{Z}\right)$.

Let us now consider the left-handed trefoil. In this case $\mathbb{H}_{\infty}=(\mathbb{Z} / 2 \mathbb{Z})^{3}, \mathbb{H}_{1}=$ $(\mathbb{Z} / 2 \mathbb{Z})^{5}$ while $\mathbb{H}_{0}=(\mathbb{Z} / 2 \mathbb{Z})^{4}$. We may compute the maps $\phi, \bar{\phi}, \psi$ and $\bar{\psi}$ as follows:

$$
\begin{aligned}
& \phi=\left(\begin{array}{lllll}
1 & 0 & 0 & 0 & 0 \\
0 & 1 & 0 & 0 & 0 \\
0 & 0 & 0 & 0 & 0
\end{array}\right), \quad \bar{\phi}=\left(\begin{array}{lllll}
0 & 0 & 0 & 0 & 0 \\
0 & 0 & 0 & 1 & 0 \\
0 & 0 & 0 & 0 & 1
\end{array}\right), \\
& \psi=\left(\begin{array}{cccc}
1 & 0 & 0 & 0 \\
0 & 1 & 0 & 0 \\
0 & 0 & 0 & 1 \\
0 & 0 & 0 & 0 \\
0 & 0 & 0 & 0
\end{array}\right), \quad \bar{\psi}=\left(\begin{array}{llll}
0 & 0 & 0 & 0 \\
0 & 0 & 0 & 0 \\
1 & 0 & 0 & 0 \\
0 & 0 & 1 & 0 \\
0 & 0 & 0 & 1
\end{array}\right) .
\end{aligned}
$$

If $q \geq p>0$, and $s=q-p$, we may form the matrix $D$ as before, and observe that the rank of this $(3 q) \times(5 s+4 p)$ matrix is $3 q$. Thus the size of the homology group for this complex would be $(5 s+4 p)+(3 q)-2 \times(3 q)=2 q-p$. If instead we have $p>q>0$, the matrix $D$ will be a $(3 q+5 r) \times(4 p)$ matrix, where $r=p-q$. The rank of this matrix may be computed as $r+3 p$. Thus the homology group of the complex is of rank $(3 q+5 r+4 p)-(6 p+2 r)=p$. This implies that $\widehat{\mathrm{HF}}\left(S_{p / q}^{3}\left(T^{\prime}\right) ; \mathbb{Z} / 2 \mathbb{Z}\right)$ is isomorphic to $(\mathbb{Z} / 2 \mathbb{Z})^{p}$ if $p \geq q$ and is isomorphic to $(\mathbb{Z} / 2 \mathbb{Z})^{2 q-p}$ if $q>p$. This completes our computation for positive rational surgery on both trefoils, using surgery formulas presented here. 


\section{References}

[1] E Eftekhary, Floer homology and existence of incompressible tori in homology spheres arXiv:math.GT/0807.2326

[2] E Eftekhary, Heegaard Floer homology and knot surgery arXiv:math. GT/0603171

[3] E Eftekhary, Heegaard Floer homology and splicing knot complements arXiv: math.GT/0802.3623

[4] E Eftekhary, A note on combinatorial splicing formulas in Heegaard Floer homology arXiv:math.GT/0804.1196

[5] E Eftekhary, A note on the rank of Heegaard Floer homology arXiv: math.GT/0803.0105

[6] E Eftekhary, Longitude Floer homology and the Whitehead double, Algebr. Geom. Topol. 5 (2005) 1389-1418 MR2171814

[7] C Manolescu, P Ozsváth, S Sarkar, A combinatorial description of knot Floer homology, Ann. of Math. (2) 169 (2009) 633-660 MR2480614

[8] C Manolescu, P Ozsváth, Z Szabó, D Thurston, On combinatorial link Floer homology, Geom. Topol. 11 (2007) 2339-2412 MR2372850

[9] P Ozsváth, A Stipsicz, Contact surgeries and the transverse invariant in knot Floer homology arXiv:math.GT/0803.1252

[10] P Ozsváth, Z Szabó, Knot Floer homology and rational surgeries arXiv: math.GT/0504404

[11] P Ozsváth, Z Szabó, Holomorphic disks and topological invariants for closed threemanifolds, Ann. of Math. (2) 159 (2004) 1027-1158 MR2113019

[12] PS Ozsváth, Z Szabó, Knot Floer homology and integer surgeries, Algebr. Geom. Topol. 8 (2008) 101-153 MR2377279

[13] S Sarkar, Maslov index of holomorphic triangles arXiv:math.GT/0609673

[14] S Sarkar, J Wang, A combinatorial description of some Heegaard Floer homologies arXiv:math.GT/0607777

School of Mathematics, Institute for Research in Fundamental Sciences (IPM) PO Box 19395-5746, Tehran, Iran

eaman@ipm.ir

Received: 25 February 2008 Revised: 16 September 2009 